\title{
TENDÊNCIA E PERFIL DA MORBIMORTALIDADE POR INFARTO AGUDO DO MIOCÁRDIO NO BRASIL
}

\section{ARTIGO ORIGINAL}

MEIRELES, Antônio Alexandre Valente ${ }^{1}$, SILVA FILHO, Francisco Pereira da ${ }^{2}$, MARTINS, Rafael Simplício ${ }^{3}$, MORAES, Thelio Ferreira Magno de ${ }^{4}$, ARAÚJO, Maria Helena Mendonça de ${ }^{5}$

MEIRELES, Antônio Alexandre Valente. Et al. Tendência e perfil da morbimortalidade por infarto agudo do miocárdio no Brasil. Revista Científica Multidisciplinar Núcleo do Conhecimento. Ano 06, Ed. 09, Vol. 04, pp. 16-31. Setembro de 2021. ISSN: 2448-0959, Link de acesso: https://www.nucleodoconhecimento.com.br/saude/perfil-da-morbimortalidade, DOI: 10.32749/nucleodoconhecimento.com.br/saude/perfil-da-morbimortalidade

\section{RESUMO}

Objetivo: Analisar a tendência e traçar o perfil epidemiológico da morbimortalidade por Infarto Agudo do Miocárdio (IAM) no Brasil, entre 2010 e 2019. Pergunta Problema: Qual a tendência da prevalência da morbimortalidade por Infarto Agudo do Miocárdio no Brasil? Metodologia: Estudo descritivo, do tipo série temporal, com dados obtidos através do Sistema de Informações sobre Mortalidade (SIM) e do Sistema de Internações Hospitalares $(\mathrm{SIH})$. Foram calculados coeficientes de incidência de internações e de mortalidade, de acordo com projeções do Instituto Brasileiro de Geografia e Estatística sendo incorporados no software SPSS 20.0 para determinação de tendências temporais por regressão linear de Prais-Winstein, com cálculo da Variação Percentual Anual (VPA) e Intervalo de Confiança de 95\%, e

\footnotetext{
${ }^{1}$ Acadêmico de Medicina.

2 Universidade Federal do Amapá (UNIFAP).

${ }^{3}$ Acadêmico de Medicina.

${ }^{4}$ Acadêmico de Medicina.

${ }^{5}$ Mestre em Ciências da Saúde. Especialização em Saúde da Família. Especialização em Medicina do Trabalho. Especialização em Saúde do Trabalhador e Ecologia Humana.
}

RC: 97161

Disponível em: https://www.nucleodoconhecimento.com.br/saude/perfil-damorbimortalidade 
valor de $p<0,05$. Resultados: Foram registradas 992.504 internações por IAM no país, com predomínio bruto no Sudeste, e ajustado no Sul. Observou-se maior prevalência na cor/raça branca e sexo masculino. Foram 113.907 os óbitos, com coeficiente médio de mortalidade de 5,61, com predomínio absoluto no Sudeste, e ajustado no Sul, percebendo-se predominância na cor/raça branca e sexo masculino. Percebe-se morbidade mais precoce em homens e mortalidade mais tardia nas mulheres. A tendência de prevalência para incidência de internações foi crescente, com VPA $=+1,62 \%$ (IC95\% 0,05; 3,23; $p=0,037)$, assim como para mortalidade, com VPA $=+5,20$ (IC95\% 4,65; 5,75; $p<0,0001)$. A tendência crescente estatisticamente significativa foi evidenciada em todas as regiões do país.

Palavras-chave: Infarto Agudo do Miocárdio, Mortalidade, Epidemiologia, Hospitalização.

\section{INTRODUÇÃO}

As doenças cardiovasculares, dentre eles o Infarto Agudo do Miocárdio (IAM), possuem um fator importante nos indicadores de morbimortalidade no Brasil, sendo a primeira causa de mortalidade proporcional no país desde a década de 1960 . Em 1998, 25\% dos óbitos na população masculina e $31,3 \%$ na feminina foram por doenças cardiovasculares, sendo, portanto, um problema de saúde pública no território nacional (BAENA; LUHM; COSTANTINI, 2012).

Apesar das doenças cardiovasculares estarem relacionadas ao desenvolvimento econômico, o Brasil encontra-se entre as maiores taxas de mortalidade do mundo (183,3/100.000), sendo semelhante à de países como a China e do Leste Europeu (MALTA et al., 2014; MORAN et al., 2014). Devido ao caráter agudo da doença, grande parte das mortes por Infarto Agudo do Miocárdio (IAM) ocorrem nas primeiras horas de manifestação clínica, sendo 40 a $65 \%$ na primeira hora e, aproximadamente, $80 \%$ nas primeiras 24 horas (BRAUNWALD; ZIPES; LIBBY, 2001; KANNEL; CUPPLES; D'AGOSTINO, 1987; TUNSTALL-PEDOE et al., 1994). 
O IAM é caracterizado por um processo isquêmico abrupto que resulta na morte dos cardiomiócitos, ocasionados por um desequilíbrio entre oferta e demanda de nutrientes ao tecido, dentre eles o oxigênio. Isso decorre da obstrução do fluxo coronariano, podendo ser transitória ou permanente, gerada por trombose e/ou vasoespasmo sobre uma placa aterosclerótica que migra impedindo a passagem do sangue oxigenado para o músculo cardíaco. Além do risco elevado de mortalidade, o indivíduo pode ser acometido por sequelas, gerando repercussões físicas e psicossociais (DAVIES, 2000; SILVEIRA et al., 2016)

Os principais fatores de risco para o desenvolvimento do IAM são a faixa etária, o sexo masculino, a hereditariedade, a hipercolesterolemia, a hipertensão arterial, o tabagismo, o sedentarismo e obesidade, fatores que estão em crescente na população brasileira (IBGE, 2011). A prevalência de IAM foi significativamente maior nos que tinham maior Índice de Massa Corporal (IMC), nos que consomem maior quantidade de embutidos e prevalência menor entre os que possuem, já há mais de um ano, o hábito de caminhar (SILVA; SOUSA; SCHARGODSKY, 1998)

O músculo cardíaco sofre progressivo dano celular, com a evolução do tempo de isquemia. No primeiro momento, ocorre o predomínio de distúrbios eletrolíticos, seguido alterações morfológicas reversíveis e, por fim, danos celulares irreversíveis. Nesse sentido, conforme o acometimento dos cardiomiócitos as manifestações clínicas são bastantes varáveis indo desde a angina instável e infarto sem supra até o infarto com supradesnível do segmento ST (BRAUNWALD; ZIPES; LIBBY, 2001)

O diagnóstico é realizado por meio da história clínica, eletrocardiograma e marcadores de isquemia miocárdica. A manifestação clínica típica é caracterizada por dor precordial em aperto no hemitórax esquerdo, irradiada para o membro superior esquerdo e/ou mandíbula, de forte intensidade e prolongada, sem alívio com o repouso ou nitratos sublinguais. É o exame mais importante no diagnóstico do IAM (BRAUNWALD; ZIPES; LIBBY, 2001). O eletrocardiograma deve ser realizado em todo paciente com suspeita de SCA em até 10 minutos da admissão hospitalar. O supradesnível do segmento $\mathrm{ST}>1 \mathrm{~mm}$ em duas derivações contíguas determina o

RC: 97161

Disponível em: https://www.nucleodoconhecimento.com.br/saude/perfil-damorbimortalidade 
diagnóstico e correlaciona-se com a topografia do infarto (ZIMETBAUM; JOSEPHSON, 1998). Os marcadores bioquímicos são encontrados na corrente sanguínea após o rompimento da membrana celular. Na síndrome coronariana aguda, deve-se realizar a coleta dos marcadores troponina e CK-MB para auxiliar no diagnóstico e no prognóstico do paciente, não necessitando do seu resultado para iniciar o esquema terapêutico (AVEZUM JUNIOR et al., 2015; RYAN et al., 1999).

O tratamento do IAM consiste na manutenção do suporte de oxigênio e na utilização de opioides (morfina) para o alívio da dor diminuindo o consumo de oxigênio pelo miocárdio isquêmico, provocado pela ativação do sistema nervoso simpático. O uso de Nitratos é útil na reversão de eventual espasmo e/ou para alívio da dor anginosa. O Ácido acetilsalicílico é um importante antiagregante plaquetário utilizado que confere significativa redução da morbimortalidade. Além disso, a utilização de Clopidrogrel, anticoagulamentes e betabloqueadores pode ser realizada. O tratamento efetivo para o infarto com supra de ST é a recanalização da artéria acometida por meio da Intervenção coronária percutânea primária, e dever ser realizado o mais precocemente a fim de minimizar os danos ao miocárdico e melhorar o prognóstico do paciente (AVEZUM JUNIOR et al., 2015; RYAN et al., 1996; VOGEL et al., 2019).

Isto posto, considerando a importância da compreensão das peculiaridades epidemiológicas para a construção de políticas públicas exequíveis e para a delimitação do panorama e da evolução da doença a nível nacional, o objetivo do estudo é analisar a tendência e traçar o perfil epidemiológico da morbimortalidade por Infarto Agudo do Miocárdio (IAM) no Brasil.

\section{METODOLOGIA}

Trata-se de um estudo descritivo, retrospectivo, do tipo série temporal, que objetivou analisar a tendência e delimitar o perfil epidemiológico da morbimortalidade por IAM no período de janeiro de 2010 a dezembro de 2019. 
Os dados foram coletados através do Sistema de Informações sobre Mortalidade (SIM) e do Sistema de Internações Hospitalares $(\mathrm{SIH})$, disponíveis na plataforma de acesso público do DATASUS (www2.datasus.gov.br). A amostra foi determinada conforme variáveis selecionadas por capítulo IX, de doenças do aparelho circulatório, do CID-10, para internações e óbitos por Infarto Agudo do Miocárdio, a saber: Regiões (Norte, Nordeste, Sudeste, Centro-oeste e Sul), Faixa etária, Sexo, Raça/cor.

Após a coleta de dados, estes foram armazenados em planilhas no Microsoft Office Excel, versão 2017, onde foram calculados os coeficientes de incidência de internações $(\mathrm{Cl})$ e de mortalidade $(\mathrm{CM})$ por cem mil habitantes, utilizando projeções populacionais do Instituto Brasileiro de Geografia e Estatística (IBGE), no intuito de se obter as estatísticas ajustadas de morbidade e mortalidade de cada região e do país. Os coeficientes foram calculados segundo as fórmulas abaixo:

$$
\mathrm{CI}=\frac{\text { número de internações novas no ano }}{\text { população no ano }} \times 100.000 ; \mathrm{CM}=\frac{\text { número de óbitos no ano }}{\text { população no ano }} \times 100.000
$$

Após o cálculo dos coeficientes anuais, os dados foram importados para o software estatístico SPSS versão 20.0. Para cada variável quantitativa realizou-se o teste de normalidade de Kolgomorov-Smirnov, apresentando todas as variáveis distribuição normal. Para determinação de tendência, utilizou-se, a priori, regressão linear com teste de Durbin-Watson para se estimar a existência de autocorrelação serial entre os dados da amostra, fenômeno possível em estudos epidemiológicos que se utilizam de amostras populacionais, o que diminuiria a precisão do teste e iria de encontro ao pré-requisito de independência dos resíduos exigido pela análise de regressão. A regressão linear temporal se dá por meio da previsão do comportamento de uma variável a partir de outra, nesse caso, foi utilizado o ano como variável independente e os coeficientes como variáveis dependentes. A fórmula abaixo demonstra a função linear simples que se baseia a regressão, de modo que bo é valor do intercepto e $b_{1}$ o valor do coeficiente de regressão ou 
coeficiente angular da reta, pelo qual se avalia seu comportamento crescente ou

decrescente.

$$
\mathrm{y}=\hat{\beta}_{0}+\hat{\beta}_{1} \mathrm{x}
$$

No intuito de evitar erros do tipo 2, em que se admite como verdadeira a hipótese nula, quando esta é falsa, de modo que seria admitida a significância na tendência de uma série, quando esta não existe, utilizou-se a regressão linear pelo método de Prais-Winsten, útil quando se objetiva analisar uma série com intervalo temporal menor. Dessa forma, deu-se a logaritmização dos coeficientes para reduzir a heterogeneidade de variância dos resíduos, seguindo-se de nova regressão linear. Realizou-se o cálculo da Variação Percentual Anual (APC) e intervalo de confiança de $95 \%$ (IC95\%) segundo preconizado por Antunes e Cardoso (ANTUNES; CARDOSO, 2015).

$$
\begin{gathered}
\mathrm{APC}=\left[-1+10^{\mathrm{b} 1}\right] \cdot 100 \% \\
\mathrm{IC}_{95 \%}=\left[-1+10^{\mathrm{b} 1 \mathrm{~min}} \cdot\right] \cdot 100 \% ;\left[-1+10^{\mathrm{b} 1 \mathrm{máx}}\right] \cdot 100 \%
\end{gathered}
$$

Foi utilizado o $R^{2}$ (coeficiente de determinação) para se avaliar a aderência ao modelo, avaliando-se, também, a significância do teste $F$ da análise de variância (ANOVA), com valor de $\mathrm{p}<0,05$.

O presente estudo utiliza dados secundários, os quais não possibilitam qualquer identificação dos pacientes, disponíveis em plataforma online de acesso livre do Ministério da Saúde. Dessa forma, o estudo seguiu os critérios para pesquisa das resoluções de número 466/2012 e 510/2016 do Conselho Nacional de Ética em Pesquisa do Brasil.

\section{RESULTADOS E DISCUSSÃO}

Registrou-se, no período analisado, 992.504 internações por Infarto Agudo do Miocárdio no Brasil. O coeficiente médio de incidência foi de 48,82 internações por

RC: 97161

Disponível em: https://www.nucleodoconhecimento.com.br/saude/perfil-damorbimortalidade 
cem mil habitantes, de modo que a região Sudeste apresentou o maior número bruto de casos, 499.943 (50,37\%), sendo seguida pela região Sul, 196.921 (19,85\%), pela Nordeste, 193.297 (19,48\%\%), Centro-oeste, 62.173 (6,26\%), e Norte, 40.170 (4,04\%). A análise dos coeficientes de incidência médios demonstrou padrão diferenciado, de modo que Sul $(67,81)$ apresentou maior incidência de internações, seguido pelo Sudeste $(58,43)$, Centro-oeste $(40,46)$, Nordeste $(34,64)$, e Norte $(22,99)$. A evolução temporal da incidência em cada região pode ser analisada no Gráfico 1.

A maior incidência, nas regiões Sul e Sudeste, é provavelmente influenciada pelo estresse como fator determinante de risco, haja vista que a região possui o maior nível de desenvolvimento do país, logo, possui fatores desencadeantes de estresse inerentes ao alto nível infraestrutural (MOREIRA et al., 2018), bem como uma elevação de exposição a outros fatores de risco como, sedentarismo, consumo de insumos industrializados e de gordura, redução de consumo de alimentos naturais e obesidade, além das disparidades socioeconômicas e dos meios de acesso a serviços de saúde (DOS SANTOS et al., 2018). 
Gráfico 1. Evolução Temporal da incidência de internações por Infarto Agudo do Miocárdio no Brasil e nas 5 regiões.

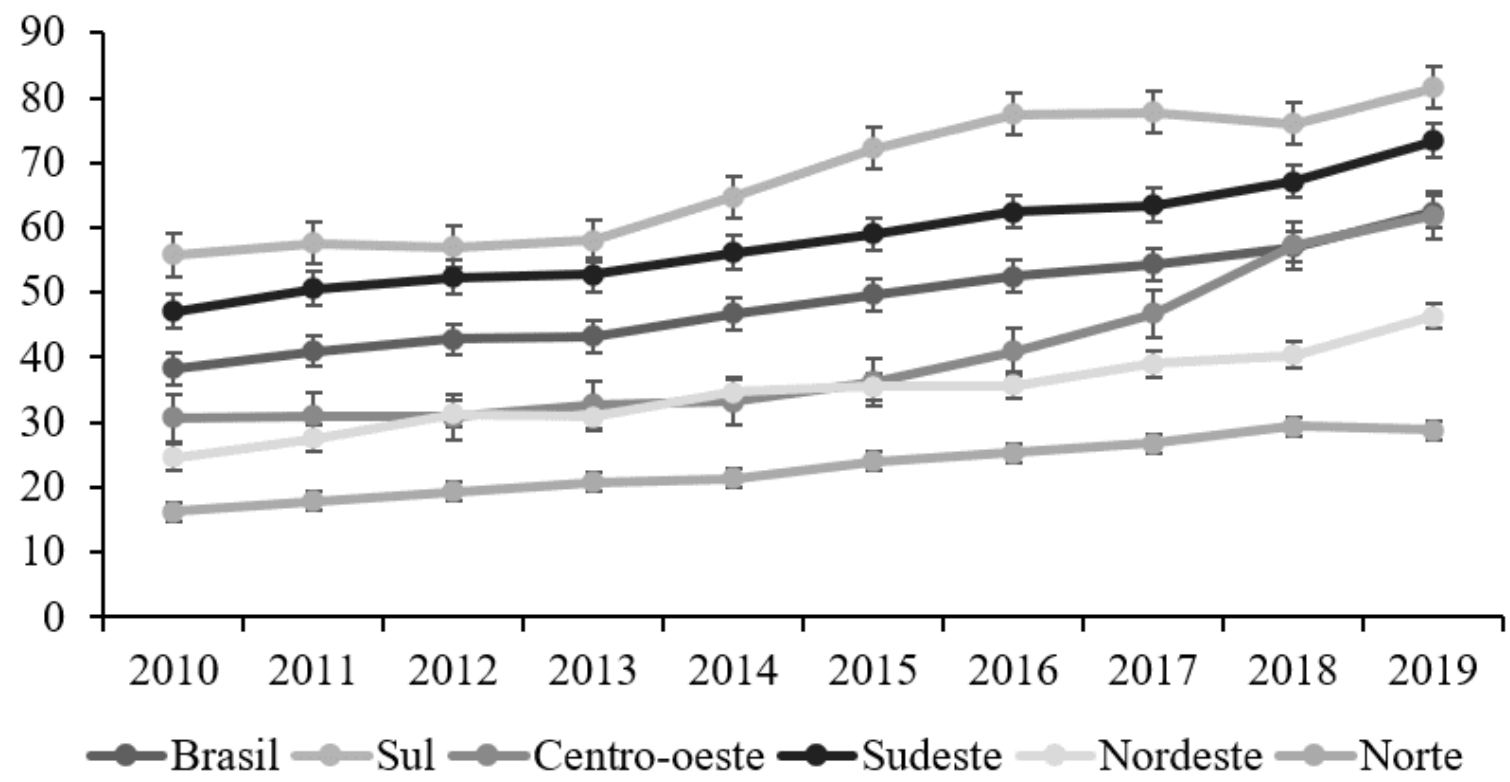

Fonte: Produção dos autores a partir de dados do Sistema de Informações Hospitalares (SIH).

A tendência da incidência demonstrou-se crescente para o Brasil, caráter presente em todas as regiões, com significância estatística. A variação percentual anual para o país foi de $+1,62 \%$ (IC $95 \% 0,05 ; 3,23 ; p=0,037$ ), as variações para as demais regiões são demonstradas na Tabela 1. Esses dados ressaltam a elevação acelerada das doenças cardiovasculares, como um todo, em outros países em desenvolvimento (VIANA; DE OLIVEIRA, 2017).

A transição demográfica, provavelmente, é a grande responsável por tal situação, visto que há 1 século atrás, as doenças infecciosas e a desnutrição eram as principais responsáveis pelos óbitos no mundo, contudo, a partir da maior exposição da população dos grandes centros a um estilo de vida com inúmeros fatores de risco já supracitados, as doenças crônicas - liderada pelas doenças cardiovasculares (DCV) - vem consolidando-se com as maiores estatísticas de morbimortalide no

RC: 97161

Disponível em: https://www.nucleodoconhecimento.com.br/saude/perfil-damorbimortalidade 
mundo. Em países desenvolvidos, os quais já se encontram em fases mais avançadas da transição epidemiológica, observa-se a queda da incidência de IAM, fenômeno que ainda não observado em países em desenvolvimento, como o Brasil, que ainda enfrentam fases iniciais dessa transição, com diminuição das doenças infectocontagiosas, e aumento das DCV (RUFF; BRAUNWALD, 2011; VOGEL et al., 2019).

Tabela 1. Tendência de incidência de internações e de mortalidade para Infarto Agudo do Miocárdio no Brasil e nas Regiões brasileiras.

\begin{tabular}{|c|c|c|c|c|c|c|}
\hline Local & Variável & VPA $^{1}$ & $\mathrm{IC}_{95 \%}{ }^{2}$ & $p^{3}$ & $\mathrm{R}^{24}$ & Tendência \\
\hline \multirow[t]{2}{*}{ Norte } & $\mathrm{Cl}^{5}$ & 6,91 & 6,$35 ; 7,46$ & $<0,0001$ & 0,906 & Crescente \\
\hline & $\mathrm{CM}^{6}$ & 6,91 & 6,$35 ; 7,46$ & $<0,0001$ & 0,989 & Crescente \\
\hline \multirow[t]{2}{*}{ Nordeste } & $\mathrm{Cl}$ & 4,23 & 2,$08 ; 6,43$ & 0,003 & 0,73 & Crescente \\
\hline & $\mathrm{CM}$ & 6,17 & 5,$07 ; 7,28$ & $<0,0001$ & 0,959 & Crescente \\
\hline \multirow[t]{2}{*}{ Sudeste } & $\mathrm{Cl}$ & 4,23 & 2,$62 ; 5,87$ & $<0,0001$ & 0,851 & Crescente \\
\hline & $\mathrm{CM}$ & 4,71 & 4,$17 ; 5,26$ & $<0,0001$ & 0,983 & Crescente \\
\hline \multirow{2}{*}{$\begin{array}{l}\text { Centro- } \\
\text { oeste }\end{array}$} & $\mathrm{Cl}$ & 1,16 & 0,$63 ; 1,69$ & 0,003 & 0,745 & Crescente \\
\hline & $\mathrm{CM}$ & 8,14 & 4,$27 ; 12,16$ & 0,002 & 0,777 & Crescente \\
\hline \multirow[t]{2}{*}{ Sul } & $\mathrm{Cl}$ & 3,28 & 2,$74 ; 3,82$ & $<0,0001$ & 0,985 & Crescente \\
\hline & $\mathrm{CM}$ & 4,71 & 3,$09 ; 6,36$ & 0,001 & 0,834 & Crescente \\
\hline \multirow[t]{2}{*}{ Brasil } & $\mathrm{Cl}$ & 1,62 & 0,$05 ; 3,23$ & 0,037 & 0,487 & Crescente \\
\hline & $\mathrm{CM}$ & 5,20 & 4,$65 ; 5,75$ & $<0,0001$ & 0,991 & Crescente \\
\hline & & & & & & \\
\hline & & & & & & \\
\hline
\end{tabular}

IVPA: variação percentual anual; ${ }^{2} C_{95 \%}$ : intervalo de $95 \%$ de confiança; ${ }^{3} p$ : valor de $p$ da regressão; ${ }^{4} R^{2}$ : ajuste do modelo; $C / 5$ : coeficiente de incidência de internação; $C M^{6}$ : coeficiente de mortalidade. 
No que tange às variáveis sociodemográficas, a cor/raça mais prevalente, quando se excluiu os "sem informação"( $n=281.494)$, foi a branca, com 401.639 casos $(56,49 \%)$, seguido pela parda em números absolutos, 268.192 (37,72\%), preta, 31605 (4,45\%), amarela, 9.248 (1,3\%), indígena, 326 (0,04\%). Contudo, percebe-se uma heterogeneidade na distribuição de cor no país, relaciona com a própria distribuição étnica da região. Nas regiões Norte e Nordeste, a maior prevalência foi de negros (pardos e pretos), enquanto nas regiões Sudeste e Sul, foi de brancos, contrariando os estudos os quais apontam a raça negra como raça/cor mais propensa a doenças cardiovasculares. Contudo, é válido ressaltar que o critério definidor de raça/cor é por meio de autodeclaração, levando-se em consideração a escolha particular do indivíduo (MOREIRA et al., 2018).

Houve predominância quantitativa do sexo masculino, 630.303 (63,5\%) internações, em relação ao feminino, 362.201 (36,5\%), apesar de ser possível perceber uma diminuição dessa distância entre as faixas etárias, através da redução da razão de gênero (H:M), com valores de 2,11 na faixa de 0 a 19 anos, 2,18 na faixa de 50 a 59 anos e 0,93 na de 80 anos ou mais, denotando uma inversão de prevalência. A distribuição do percentual de internações por faixa etária em cada gênero é exibida na Figura 1, de modo que $30,23 \%$ dos casos em homens e $28,36 \%$ dos casos em mulheres estão agrupados na faixa de 60 a 69 anos. Tal disparidade entre sexos, pode ser explicada pela provável função protetora do estradiol conferida ao sistema cardiovascular deste gênero antes do climatério e a associação da queda abrupta desse hormônio, no período menopausal, como fator de risco cardiovascular (KAUR; SINGH, 2016; TRONCOSO et al., 2018).

Pode-se suspeitar, assim, que o acometimento por IAM na terceira idade, bem como a maior sobrevida dos pacientes refletem o crescimento da expectativa de vida nacional, bem como melhores condições de saúde. Contudo, tal ocorrência também evidencia um maior acometimento nessas faixas de idade por comorbidades e/ou fatores de risco, sendo estes não modificáveis (idade, gênero, fator hereditário) e modificáveis (etilismo, tabagismo, hipertensão arterial sistêmica, sedentarismo, entre outras), os quais influem negativamente no infarto agudo do miocárdio e nas

RC: 97161

Disponível em: https://www.nucleodoconhecimento.com.br/saude/perfil-damorbimortalidade 
doenças cardiovasculares como um todo (MESQUITA, 2018; VIANA; DE OLIVEIRA, 2017).

Figura 1. Distribuição em pirâmide etária das internações por IAM no Brasil.

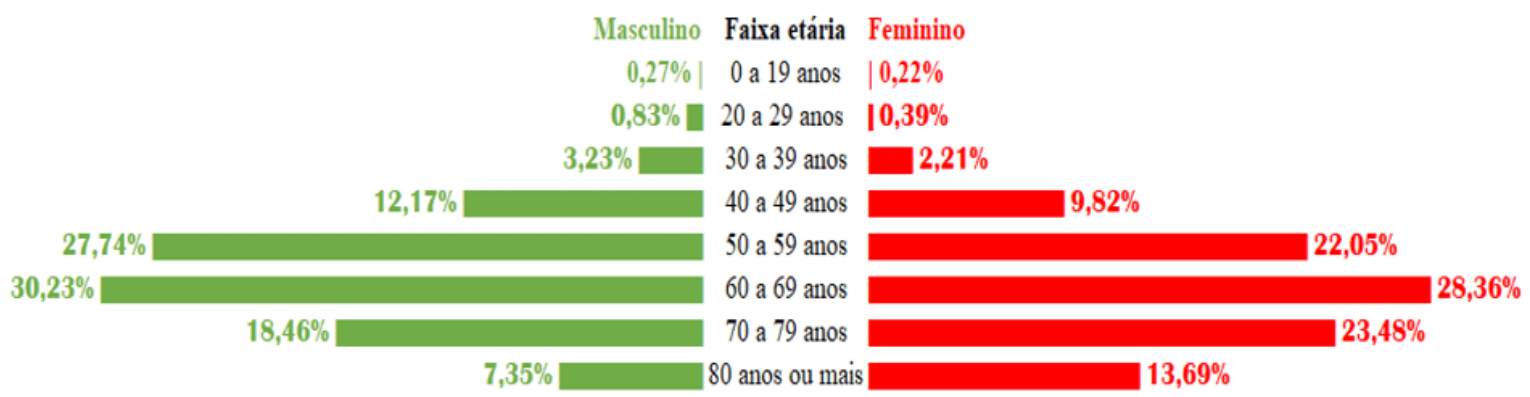

Fonte: Produção dos autores a partir de dados do Sistema de Informações Hospitalares $(\mathrm{S} / \mathrm{H})$.

Registrou-se, no período, 113. 907 óbitos por IAM no país, com coeficiente médio de mortalidade de 5,61 óbitos por cem mil habitantes, com a região Sudeste apresentando maior número absoluto, 56.069 (49,22\%), seguida pela região Nordeste, 24.359 (21,38\%), Sul, 21.212 18,62\%), Centro-oeste, 7.243 (6,35\%) e Norte, 5024 (4,41\%). O coeficiente médio de mortalidade, demonstrou maiores valores para a região Sul $(7,31)$, seguida pela Sudeste $(6,56)$, Centro-oeste $(4,71)$, Nordeste $(4,37)$, e Norte $(2,88)$. A evolução temporal desse coeficiente por região pode ser visualizada no Gráfico 2. A análise de tendência por regressão demonstrou aspecto crescente do coeficiente de mortalidade, com significância estatística, caráter presente em todas as regiões, como é demonstrado na Tabela 1. A variação percentual anual para o país foi de $+5,20 \%$ (IC95\% 4,65; 5,75; $p<0,0001$ ).

As diferenças analisadas nos coeficiente de mortalidade demonstram que apesar dos avanços aferidos em relação a melhores diagnósticos, tratamentos e ações em saúde, as diferencias sociodemográficas e econômicas diminuem o acesso de boa parte da população a essas ferramentas, interferindo negativamente na sobrevida desses indivíduos (TRONCOSO et al., 2018).

RC: 97161

Disponível em: https://www.nucleodoconhecimento.com.br/saude/perfil-damorbimortalidade 
Gráfico 2. Evolução temporal da mortalidade por Infarto Agudo do Miocárdio no Brasil e nas 5 regiões.

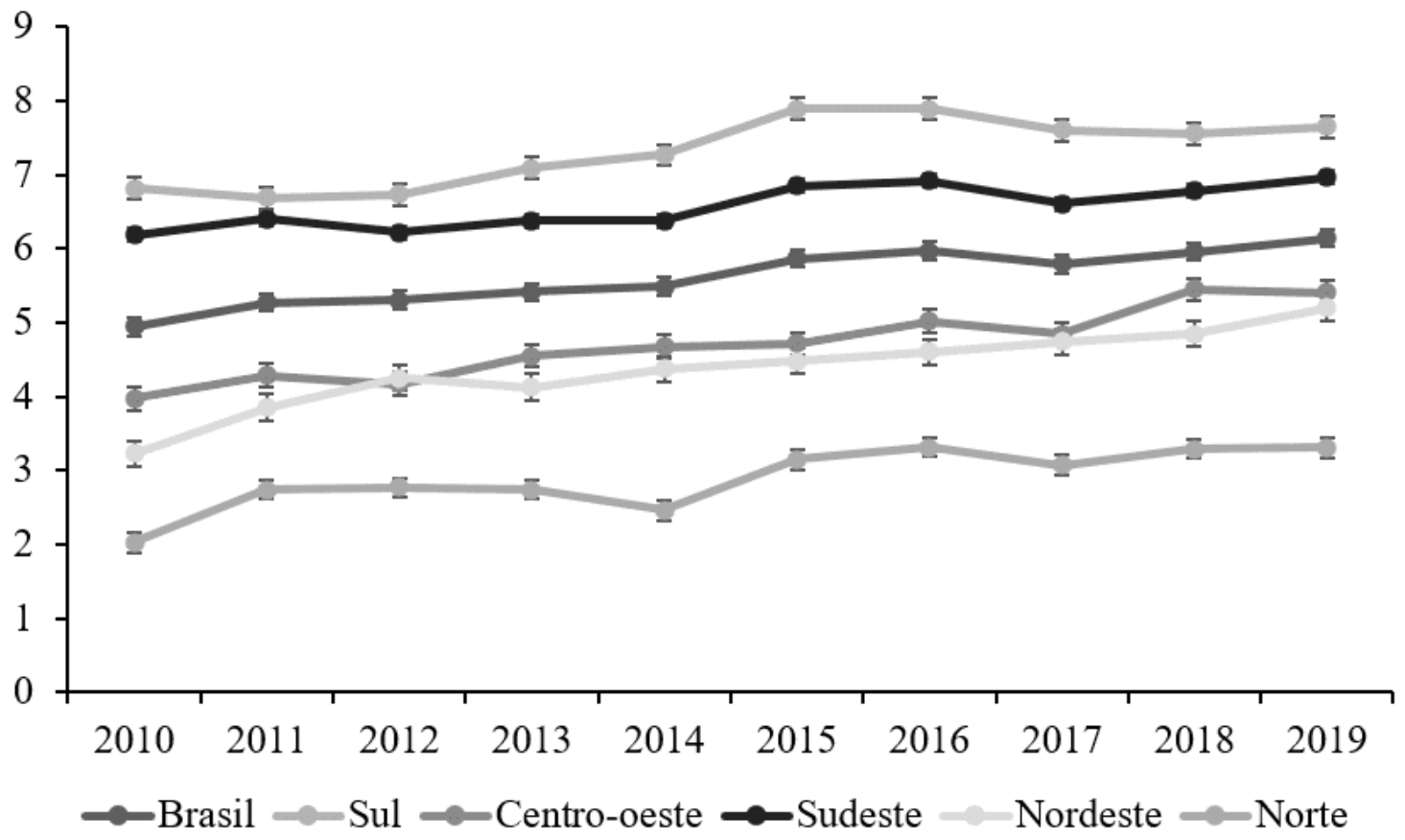

Fonte: Produção dos autores a partir de dados do Sistema de Informação de Mortalidade (SIM).

O perfil epidemiológico da mortalidade demonstrou-se pela predominância da cor branca, quando se exclui os dados da categoria "sem informação"( $n=36.158)$, com 44.756 (57,57\%) óbitos, seguido pela parda, 28470 (36,62\%), preta, 3.399 (4,38\%), amarela, 1.088 (1,39\%), e indígena, 36 (0,04\%). Contudo, é importante salientar o mesmo caráter heterogêneo observado na incidência, dependendo da região, pela distribuição étnica variada.

Quanto ao sexo, o masculino apresentou predominância quantitativa, 63.527 (55,77\%), em relação ao feminino, 50.380 (44,23\%), embora sejam observadas variações na razão de gênero (H:M), de 2,06 na faixa de 0 a 19 anos, para 1,77 para a de 50 a 59 anos, 0,84 para 80 anos ou mais, denotando inversão de mortalidade nessa faixa. A distribuição dos óbitos por faixa etária e sexo pode ser analisada na Figura 2, com $28,46 \%$ dos óbitos em homens sendo observados na faixa etária de

RC: 97161

Disponível em: https://www.nucleodoconhecimento.com.br/saude/perfil-damorbimortalidade 
60 a 69 anos, enquanto para mulheres, 30,55\% óbitos estão concentrados na faixa de 70 a 79 anos, sendo tal característica também observada em outros estudos (COELHO; RESENDE, 2010; MOREIRA et al., 2018; TRONCOSO et al., 2018). A literatura demonstra maior letalidade da doença na fase aguda para a população feminina, com maior prevalência de disfunção ventricular esquerda, além da provável exposição desigual dos fatores de risco entre os sexos, com as mulheres a presença da síndrome metabólica aumentando gradualmente no gênero, o que justificaria a inversão de prevalência observada em faixas etárias mais elevadas (DOS SANTOS et al., 2018; TRONCOSO et al., 2018).

Essa progressiva mudança nas taxas de mortalidade por IAM também são encontrados em outros países e refletem uma alteração nas exposições aos fatores de risco, bem como o aumento expectativa de vida, proporcionada pela melhoria nos diagnósticos, tratamentos e a busca por um estilo de vida mais saudável. Além disso, por tratar-se de uma doença de caráter crônico, a maior mortalidade em faixas etárias mais avançadas é, até certo ponto, esperada (DOS SANTOS et al., 2018).

Figura 2. Distribuição em pirâmide etária dos óbitos por IAM no Brasil.

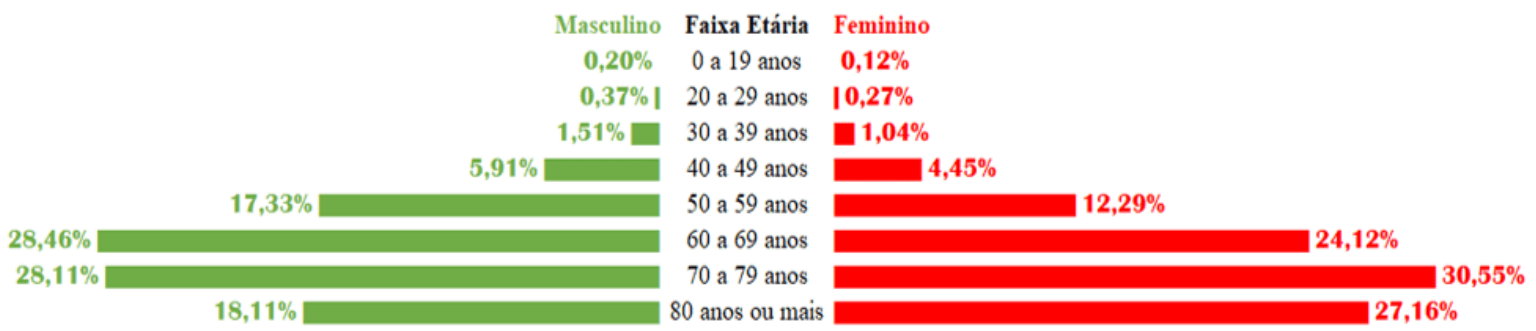

Fonte: Produção dos autores a partir de dados do Sistema de Informação de Mortalidade (SIM).

\section{CONSIDERAÇÕES FINAIS}

O presente estudo sinaliza que no âmbito das internações e mortalidade por IAM, temos maior número absoluto na região sudeste e padrão diferente quanto ao coeficiente de incidência média, predominando na região Sul. A tendência temporal

RC: 97161

Disponível em: https://www.nucleodoconhecimento.com.br/saude/perfil-damorbimortalidade 
demonstra taxa crescente anual no que tange a internações e mortalidade por IAM. No tocante a etnia, a branca é mais prevalente em ambos os casos; apesar dos dados nos chamarem atenção sobre o montante expressivo de pacientes não identificados e da heterogeneidade étnica de acordo com a região. No que diz respeito ao gênero, o masculino domina, notando-se, contudo, um padrão decrescente sobre a razão de sexos entre as faixas etárias, demostrando uma inversão de prevalência em faixas etárias maiores.

Este estudo apresenta limitações intrínsecas a estudos de caráter epidemiológico que utilizam dados secundários. Dentre os quais o viés de seleção se faz presente, já que os dados são provenientes de notificação; e de registro, que pode não ter sido feito de forma adequada. Apesar disso, técnicas de metodologia estatística foram utilizadas com o intuito de diminuir esses vieses, conferindo análises mais robustas à amostra, de forma a representar qualitativo e quantitativamente de forma mais fidedigna e verossímil a realidade epidemiológica.

Estudos como esse reiteram a necessidade de atuação sobre notificação e na aplicação clínica da medicina baseada em evidências para promover melhorias na prevenção e manejo desses grupos. Neste contexto, análise do estudo enfatiza a relevância de se conhecer o perfil de indivíduos acometidos por infarto agudo do miocárdio, bem como a epidemiologia dos pacientes que possuem um desfecho negativo desse quadro. Tal conhecimento é de extrema importância na melhor tomada de decisão clínica, além de fornecer subsídio para o direcionamento adequado das ações em saúde as quais abordam, tanto o IAM quanto as doenças cardiovasculares, na diminuição de fatores de risco modificáveis, de internações e de óbitos em um trabalho matricial a partir da prevenção primária, secundária e terciária.

\section{REFERÊNCIAS}

ANTUNES, José Leopoldo Ferreira; CARDOSO, Maria Regina Alves. Uso da análise de séries temporais em estudos epidemiológicos. Epidemiologia e Serviços de 
Saúde, vol. 24, no. 3, p. 565-576, 2015. https://doi.org/10.5123/s167949742015000300024.

AVEZUM JUNIOR, Álvaro; FELDMAN, André; CARVALHO, Antônio Carlos De Camargo; SOUSA, Antônio Carlos Sobral; MANSUR, Antônio De Pádua; BOZZA, Augusto Elias Zaffalon; FALCÃO, Breno De Alencar Araripe; FILHO, Brivaldo Markman; POLANCZYK, Carisi Anne; GUN, Carlos; SERRANO JUNIOR, Carlos Vicente; OLIVEIRA, César Cardoso De; MOREIRA, Dalmo; PRÉCOMA, Dalton Bertolim; MAGNONI, Daniel; ALBUQUERQUE, Denílson Campos De; ROMANO, Edson Renato; STEFANINI, Edson; SANTOS, Elizabete Silva Dos; ... MATHIAS JUNIOR, Wilson. V Diretriz da Sociedade Brasileira de Cardiologia sobre Tratamento do Infarto Agudo do Miocárdio com Supradesnível do Segmento ST. Arquivos Brasileiros de Cardiologia, vol. 105, no. 2, p. 1-105, 2015. https://doi.org/10.5935/abc.20150107.

BAENA, Cristina Pellegrino; LUHM, Karin Regina; COSTANTINI, Constantino Ortiz. Tendência de Mortalidade por Infarto Agudo do Miocárdio em Curitiba ( PR ) no Período de 1998 a 2009. Arquivos Brasileiro de Cardiologia, , p. 211-217, 2012. .

BRAUNWALD, Eugene; ZIPES, Douglas P; LIBBY, Peter. Braunwald: Heart Disease: A Textbook of Cardiovascular Medicine, 6th ed., Copyright $\odot 2001$ W. B. Saunders Company. [S. I.: s. n.], 2001.

COELHO, Letícia Maria; RESENDE, Elmiro Santos. Perfil dos pacientes com infarto do miocárdio, em um hospital universitário. Rev Med Minas Gerais, vol. 20, no. 3, p. 323-328, 2010. https://doi.org/10.1017/CBO9781107415324.004.

DAVIES, Michael J. The pathophysiology of acute coronary syndromes. Education in Heart, , p. 361-366, 2000 .

DOS SANTOS, Juliano; MEIRA, Karina Cardoso; CAMACHO, Amanda Rodrigues; SALVADOR, Pétala Tuani Candido de Oliveira; GUIMARÃES, Raphael Mendonça; PIERIN, Ângela Maria Geraldo; SIMÕES, Taynãna Cesar; FREIRE, Flávio Henrique 
Miranda de Araújo. Mortality due to acute myocardial infarction in Brazil and its geographical regions: Analyzing the effect of age-period-cohort. Ciencia e Saude Coletiva, vol. 23, no. 5, p. 1621-1634, 2018. https://doi.org/10.1590/141381232018235.16092016 .

IBGE. Instituto Brasileiro de Geografia e Estatística. Pesquisa de orçamentos familiares 2008-2009: análise do consumo alimentar pessoal no Brasil. 2011. Acesso em: https://biblioteca.ibge.gov.br/visualizacao/livros/liv50063.pdf

KANNEL, William B.; CUPPLES, L. Adrienne; D'AGOSTINO, Ralph B. Sudden death risk in overt coronary heart disease: The Framingham Study. American Heart Journal, vol. 113, no. 3, p. 799-804, 1987. https://doi.org/10.1016/00028703(87)90722-8.

KAUR, Amanpreet; SINGH, Jagdeep. Prevalence of risk factors in patients with myocardial infarction. Indian Journal of Public Health Research and Development, vol. 7, no. 1, p. 242-245, 2016. https://doi.org/10.5958/09765506.2016.00046.2.

MALTA, Deborah Carvalho; MOURA, Lenildo de; PRADO, Rogério Ruscitto do; ESCALANTE, Juan Cortez; SCHMIDT, Maria Inês; DUNCAN, Bruce Bartholow. Mortalidade por doenças crônicas não transmissíveis no Brasil e suas regiões, 2000 a 2011. Epidemiologia e Serviços de Saúde, vol. 23, no. 4, p. 599-608, 2014. https://doi.org/10.5123/s1679-49742014000400002.

MESQUITA, Claudio Tinoco. Relação entre Fatores Sociais e Doenças Cardiovasculares. International Journal Of Cardiovascular Sciences, vol. 31, no. 2, p. 87-89, 2018. Available at: http://www.scielo.br/pdf/ijcs/v31n2/pt_2359-4802-ijcs31-02-0087.pdf.

MORAN, Andrew E; FOROUZANFAR, Mohammad H; ROTH, Gregory A; MENSAH, George A; EZZATI, Majid; MURRAY, Christopher J L. Temporal Trends in Ischemic Heart Disease Mortality in 21 World Regions, 1980 to 2010 The Global Burden of

RC: 97161

Disponível em: https://www.nucleodoconhecimento.com.br/saude/perfil-damorbimortalidade 
Disease 2010 Study. Circulation, vol. 129, no. 14, p. 1483-1492, 2014. https://doi.org/10.1161/CIRCULATIONAHA.113.004042.Temporal.

MOREIRA, Márcia Adriana Dias Meirelles; CUNHA, Mônica Lorena Dias Meirelles da; CAVALCANTI NETO, Francisco de Assis; SOUTO, José Gomes; MEDEIROS JÚNIOR, Ivson José Almeida. Perfil dos pacientes atendidos por infarto agudo do miocárdio. Rev. Soc. Bras. Clín. Méd, vol. 16, no. 4, p. 212-214, 2018. Available at: http://fi-admin.bvsalud.org/document/view/w8rk9.

RUFF, Christian T.; BRAUNWALD, Eugene. The evolving epidemiology of acute coronary syndromes. Nature Reviews Cardiology, vol. 8, no. 3, p. 140-147, 2011. DOI 10.1038/nrcardio.2010.199.

Available at: http://dx.doi.org/10.1038/nrcardio.2010.199.

RYAN, Thomas J.; ANDERSON, Jeffrey L.; ANTMAN, Elliott M.; BRANIFF, Blaine A.; BROOKS, Neil H.; CALIFF, Robert M.; HILLIS, L. David; HIRATZKA, Loren F.; RAPAPORT, Elliot; RIEGEL, Barbara J.; RUSSELL, Richard O.; SMITH, Earl E.; WEAVER, W. Douglas; RITCHIE, James L.; CHEITLIN, Melvin D.; EAGLE, Kim A.; GARDNER, Timothy J.; GARSON, Arthur; GIBBONS, Raymond J.; ... O'ROURKE, Robert A. ACC/AHA Guidelines for the Management of Patients with Acute Myocardial Infarction. A report of the American College of Cardiology/American Heart Association Task Force on Practice Guidelines (Committee on Management of Acute Myocardial Infarction). [S. I.: s. n.], 1996. vol. 28, . https://doi.org/10.1016/S0735-1097(96)00392-0.

RYAN, Thomas J; ANTMAN, Elliott M; BROOKS, Neil H; CALIFF, Robert M; HILLIS, L.David; HIRATZKA, Loren F; RAPAPORT, Elliot; RIEGEL, Barbara; RUSSELL, Richard O; SMITH, Earl E; WEAVER, W.Douglas; GIBBONS, Raymond J; ALPERT, Joseph S; EAGLE, Kim A; GARDNER, Timothy J; GARSON, Arthur; GREGORATOS, Gabriel; RUSSELL, Richard O; RYAN, Thomas J; SMITH, Sidney C. 1999 update: ACC/AHA guidelines for the management of patients with acute 
myocardial infarction. Journal of the American College of Cardiology, vol. 34, no. 3, p. 890-911, 1999. https://doi.org/10.1016/s0735-1097(99)00351-4.

SILVA, Marco Aurélio Dias da; SOUSA, Amanda G. M. R.; SCHARGODSKY, Herman. Fatores de Risco para Infarto do Miocárdio no Brasil. Arq Bras Cardiol, vol. 71 , no. 5, p. 667-675, 1998 . .

SILVEIRA, Daniel Souto; JAEGER, Cristiano Pederneiras; HATSCHBACH, Luciano; MANENTI, Euler Roberto Fernandes. Validação do Escore TIMI de Risco para Infarto Agudo com Supradesnivelamento do Segmento ST. Int. j. cardiovasc. sci. (Impr.), vol. 29, no. 3, p. f:189-I:197, 2016. Available at: http://pt.

TRONCOSO, Luiza T; OLIVEIRA, Nathália C.C; LARANJEIRA, Nelson R. F; LEPORAES, Rômulo C. A; EIRA, Tadeu L; PINHEIRO, Vitória P. Estudo Epidemiológico Da Incidência Do Infarto Agudo Do Miocárdio Na População Brasileira. Revista Caderno de Medicina №1, vol. 1, p. 91-101, 2018. Available at: http://unifeso.edu.br/revista/index.php/cadernosdemedicinaunifeso/article/viewFile/95 $7 / 450$.

TUNSTALL-PEDOE, $\mathrm{H}$; KUULASMAA, K; AMOUYEL, P; ARVEILER, D; RAJAKANGAS, A M; PAJAK, A. Myocardial infarction and coronary deaths in the World Health Organization MONICA Project. Registration procedures, event rates, and case-fatality rates in 38 populations from 21 countries in four continents. Circulation, vol. 90, no. 1, p. 583-612, 1994. DOI 10.1161/01.cir.90.1.583. Available at: http://10.0.4.137/01.cir.90.1.583.

VIANA, Tatiane Lima; DE OLIVEIRA, Maria Luiza Carvalho. Risk factors for the development of coronary artery disease in civil construction professionals. Mundo da Saude, vol. 41, no. 2, p. 154-162, 2017. https://doi.org/10.15343/01047809.20174102154162 .

VOGEL, Birgit; CLAESSEN, Bimmer E.; ARNOLD, Suzanne V.; CHAN, Danny; COHEN, David J.; GIANNITSIS, Evangelos; GIBSON, C. Michael; GOTO, Shinya;

RC: 97161

Disponível em: https://www.nucleodoconhecimento.com.br/saude/perfil-damorbimortalidade 
KATUS, Hugo A.; KERNEIS, Mathieu; KIMURA, Takeshi; KUNADIAN, Vijay; PINTO, Duane S.; SHIOMI, Hiroki; SPERTUS, John A.; STEG, P. Gabriel; MEHRAN, Roxana. ST-segment elevation myocardial infarction. Nature Reviews Disease Primers, vol. 5, no. 1, p. 1-20, 2019. DOI 10.1038/s41572-019-0090-3. Available at: http://dx.doi.org/10.1038/s41572-019-0090-3.

ZIMETBAUM, Peter J.; JOSEPHSON, Mark E. Use of the Electrocardiogram in Acute Myocardial Infarction. Mayo Clinic Proceedings, vol. 73, no. 12, p. 1229, 1998. https://doi.org/10.4065/73.12.1229.

Enviado: Fevereiro, 2021.

Aprovado: Setembro, 2021. 